\title{
Optimization of transfection parameters for ultrasound/SonoVue microbubble-mediated hAng-1 gene delivery in vitro
}

\author{
QING ZHOU, JIN-LING CHEN, QIAN CHEN, XIAO WANG, QING DENG, BO HU and RUI-QIANG GUO \\ Department of Ultrasound Imaging, Renmin Hospital of Wuhan University, Wuhan 430060, P.R. China
}

Received May 16, 2012; Accepted September 20, 2012

DOI: $10.3892 / \mathrm{mmr} .2012 .1100$

\begin{abstract}
This study aimed to explore the effects of microbubble concentration, gene dosage, cell-microbubble mixing mode and fetal bovine serum (FBS) on gene delivery. 293T cells were transfected with SonoVue microbubbles carrying the hAng-1 gene via ultrasound irradiation. Various ultrasound exposure parameters and microbubble and DNA concentrations were investigated. In addition, FBS and the cell suspension or adherent mode was explored. Transfection efficiency and cell viability were used to determine the optimal transfection parameters. hAng-1 gene transfection efficiency gradually increased with elongation of ultrasound exposure and increasing microbubble concentration. However, if ultrasound irradiation exceeded $1.5 \mathrm{~W} / \mathrm{cm}^{2}$ and $30 \mathrm{sec}$ or the microbubble concentration was over $20 \%$, hAng-1 gene expression was significantly decreased, coupled with extensive cell death. Gene transfection levels were low under DNA concentrations less than $15 \mu \mathrm{g} / \mathrm{ml}$. Furthermore, the gene transfer rate was significantly increased under cell suspension mode; FBS had no effect on hAng-1 gene transfection. The integrity of hAng-1 DNA was not affected by ultrasonic irradiation under optimal conditions. The optimal transfection parameters for the hAng-1 gene and SonoVue microbubble were ultrasound exposure of $1.5 \mathrm{~W} / \mathrm{cm}^{2}$ and $30 \mathrm{sec}, 20 \%$ microbubbles, $15 \mu \mathrm{g} / \mathrm{ml}$ of DNA and under cell suspension mode.
\end{abstract}

\section{Introduction}

Ultrasound-mediated microbubble fragmentation technology is used to transport remedial exogenous genes or drugs into impaired organs or tissues and create biological effects. This technology has become an extremely promising gene delivery tool for myocardial ischemia gene therapy (1-4). Previous studies have mainly focused on the effects of ultrasound irradiation parameters on gene transfer efficiency (5-7), however, this study was designed to explore the effects of other parameters,

Correspondence to: Dr Rui-Qiang Guo, Department of Ultrasound Imaging, Renmin Hospital of Wuhan University, 238\# Jiefang Road, Wuhan 430060, P.R. China

E-mail: ruiqiangguo@126.com

Key words: hAng-1 gene, microbubble-mediated, ultrasound, gene delivery including microbubble concentration, gene dosage, cell-microbubble mixing mode and the presence of fetal bovine serum (FBS), and how to match these parameters to achieve good gene delivery. Furthermore, therapeutic gene integrity post ultrasound irradiation was also tested, as it is fundamental for gene expression. The ultimate aim is to provide an objective basis for future angiogenesis research of microbubble/ultrasound-mediated hAng-1 gene delivery into ischemic myocardium in vivo.

\section{Materials and methods}

Preparation of SonoVue microbubble/hAng-1 gene suspension. The eGFP-hAng-1 construct was generated by PCR with a high-fidelity thermostable DNA polymerase (Fermentas Co., Glen Burnie, MD, USA) based on the pAAVAVNGPT1 plasmid (designed and presented by Wu's Lab, College of Life Science, Wuhan University, Wuhan, China). The suspension of SonoVue microbubbles was prepared according to the manufacturer's instructions. The SonoVue microbubble was produced by Bracco Co. (Geneva, Switzerland), with a diameter of $2.5 \mu \mathrm{m}$ and coated with phospholipids on the surface. The mixture of the suspension of the hAng-1 plasmid and SonoVue microbubbles was kept at $4^{\circ} \mathrm{C}$ for 15 min with oscillation to ensure the plasmid and microbubbles were in full contact and adhered to one other. The study was approved by the ethics committee of Renmin Hospital of Wuhan University, Wuhan, China.

Determination of optimal ultrasound exposure. Human embryonic kidney 293T cells were maintained in DMEM with $10 \%$ FBS. For transfection, cells were seeded at $1 \times 10^{6}$ cells/well in 6-well plates and transfected with $10 \% \mu \mathrm{l} / \mu \mathrm{l}$ microbubble suspension containing $10 \mu \mathrm{g} / \mathrm{ml}$ hAng-1 gene via ultrasound irradiation (8-10). During transfection, the ultrasound probe was placed under the 6-well plate at a distance of 3-5 mm, and irradiation frequency was ranged from $0.5-2 \mathrm{MHz}$ with continuous wave (UGT2007 ultrasound irradiation machine, Ultrasonic Research Institute, Chongqing Medical School, China). The irradiation intensity was set to $0.5,1.0,1.5$ and $2.0 \mathrm{~W} / \mathrm{cm}^{2}$ with exposure time set to $10,20,30,60$ and $120 \mathrm{sec}$. Transfected cells were exposed to ultrasound irradiation over various time courses, and transfection efficiency and cell viabilities were used to determine the optimal ultrasound exposure parameters.

Determination of optimal microbubble concentration. To determine the optimal matching parameters between micro- 
bubble concentration and ultrasound exposure, the microbubble concentration was set to 5, 10, 20,30 and 40\% ( $\mu 1 / \mu 1)$ under the ultrasound exposure dosage of 1.5, 3.0, 4.5 and $6.0 \mathrm{~W} / \mathrm{cm}^{2} \mathrm{x}$ sec (intensity x time). Microbubble concentration was calculated as microbubble solution volume/(microbubble solution volume + reaction system volume; $\mu 1 / \mu 1)$. The plasmid DNA concentration was $10 \mu \mathrm{g} / \mathrm{ml}$. hAng-1 gene delivery rate and cell death under these conditions were used to determine the optimal microbubble concentration.

Determination of optimal plasmid DNA concentration. To determine the optimal concentration of the hAng-1 gene in cell suspension, plasmids were prepared at 2.5, 5, 10, 15, 20 and $30 \mu \mathrm{g} / \mathrm{ml}$. hAng-1 gene delivery rate and cell death under optimal microbubble conditions and ultrasound radiation parameters remained as above.

Determination of mixed mode of cells and microbubble. To determine the effect of mixed mode on transfection efficiency and cell viabilities, 293T cells and microbubbles carrying the hAng-1 gene were cultured in adherence and suspension modes. In suspension mode, the microbubbles carrying the gene were added into cultured 293T cells in 6-well plates at the moment of inoculation, followed by ultrasound irradiation. Under adherence mode, $293 \mathrm{~T}$ cells at $1 \times 10^{6} / \mathrm{ml}$ concentration were seeded in 6-well plates (total number of cells remained constant under both modes). Following adhesion, the microbubble suspension carrying the gene was added, followed by ultrasound irradiation. All other experimental parameters remained constant between the two modes.

Effect of FBS on transfection system. To determine the effect of FBS on transfection efficiency and cell viability, the transfection efficiency of $293 \mathrm{~T}$ cells, transfected with the hAng-1 plasmid at $15 \mu \mathrm{g} / \mathrm{ml}$ concentration via ultrasound irradiation with $8 \%$ FBS was compared with that in the absence of FBS. All other experimental parameters followed the above optimal conditions and remained constant between the two groups.

Control group. Cells were transfected with microbubble suspension carrying the gene hAng-1 as the experimental group, and cells were transfected with only the hAng-1 gene as the control group. All other experimental parameters remained constant.

Determination of gene transfection efficiency and cell viability. Fluorescence quantity and intensity of green fluorescent protein (GFP) under the fluorescence microscope and percentage of eGFP positive cells via flow cytometry were used to determine the transfection efficiency. Cell viability was determined by $0.4 \%$ trypan blue staining and the cell counting method. Cell morphology and structure were compared using an inverted microscope under the conditions stated.

Detection of hAng-1 mRNA. hAng-1 gene expression at the mRNA level was determined by quantitative RT-PCR. The primer sequence used for detection was designed, upstream, 5'-TGCCATTACCAGTCAGAGG-3', and downstream, 5'-CAAGCATCAAACCACCATC-3', using Primer Premier 5.0 software and the product was analyzed by $1 \%$ agarose gel electrophoresis.
Table I. Effect of ultrasonic irradiation intensity on efficiency of gene transfer and cell viability (mean $\pm \mathrm{SD})$.

\begin{tabular}{lcc}
\hline Intensity $\left(\mathrm{W} / \mathrm{cm}^{2}\right)$ & Transfection efficiency & Cell viability \\
\hline 0.5 & $5.2 \pm 1.9$ & $92.8 \pm 2.0$ \\
1.0 & $10.1 \pm 0.9$ & $88.9 \pm 2.4$ \\
1.5 & $15.5 \pm 1.0^{\mathrm{a}}$ & $83.2 \pm 2.2$ \\
2.0 & $14.2 \pm 2.3^{\mathrm{a}}$ & $68.1 \pm 4.6^{\mathrm{b}}$ \\
\hline
\end{tabular}

Transfection efficiency compared with $0.5,1.0 \mathrm{~W} / \mathrm{cm}^{2},{ }^{\mathrm{a}} \mathrm{P}<0.01$. Cell viability compared with $0.5,1.0$ and $1.5 \mathrm{~W} / \mathrm{cm}^{2},{ }^{\mathrm{b}} \mathrm{P}<0.01$.

Table II. Effect of ultrasonic irradiation duration on efficiency of gene transfer and cell viability (mean $\pm \mathrm{SD})$.

\begin{tabular}{lcc}
\hline Duration $(\mathrm{sec})$ & Transfection efficiency & Cell viability \\
\hline 10 & $2.7 \pm 0.7$ & $91.7 \pm 2.1$ \\
20 & $9.5 \pm 0.4$ & $84.9 \pm 1.9$ \\
30 & $15.9 \pm 0.9^{\mathrm{a}}$ & $82.6 \pm 2.6$ \\
60 & $11.8 \pm 1.2$ & $75.0 \pm 2.1^{\mathrm{b}}$ \\
120 & $8.7 \pm 1.0$ & $64.6 \pm 3.8^{\mathrm{b}}$ \\
\hline
\end{tabular}

Transfection efficiency compared with 10,20,60 and $120 \mathrm{sec}$; ${ }^{\mathrm{a} P}<0.01$. Cell viability compared with $10,20,30,60$ or $120 \mathrm{sec}$; ${ }^{\text {b }} \mathrm{P}<0.01$.

Detection of hAng-1 protein. hAng-1 gene expression at the protein level was determined by western blot analysis. Cell lysis was performed $48 \mathrm{~h}$ following hAng-1 gene transfection and hAng-1 protein was detected using a primary antibody against hAng-1, purchased from Abcam Co. (Cambridge, MA, USA; Angiopoietin 1 antibody, ab8451).

Analysis of DNA integrity following microbubble-mediated gene transfer. To determine the integrity of DNA after ultrasound irradiation, the hAng-1 solution was analyzed by agarose gel electrophoresis following ultrasound irradiation. The result was compared with that of the hAng-1 gene solution wtihout ultrasonic irradiation.

Statistical analysis. SPSS 11.0 was used for statistical analysis. Data are expressed as the mean $\pm \mathrm{SD}$, and one-way ANOVA and post hoc tests were used to analyze the significance of hAng-1 gene transfection efficiency and cell viability under the above various experimental conditions.

\section{Results}

Ultrasound exposure parameters. Gene transfection efficiency increased with enhancement of irradiation intensity and extension of irradiation time. However, further increases were not obtained at irradiation intensity and duration beyond $1.5 \mathrm{~W} / \mathrm{cm}^{2}$ and $30 \mathrm{sec}$. In addition, cell viability decreased significantly beyond these settings (Tables I and II). Taking into account the transfection efficiency and cell survival rate, $1.5 \mathrm{~W} / \mathrm{cm}^{2}$ and $30 \mathrm{sec}$ were set as optimal conditions in our experiments. 


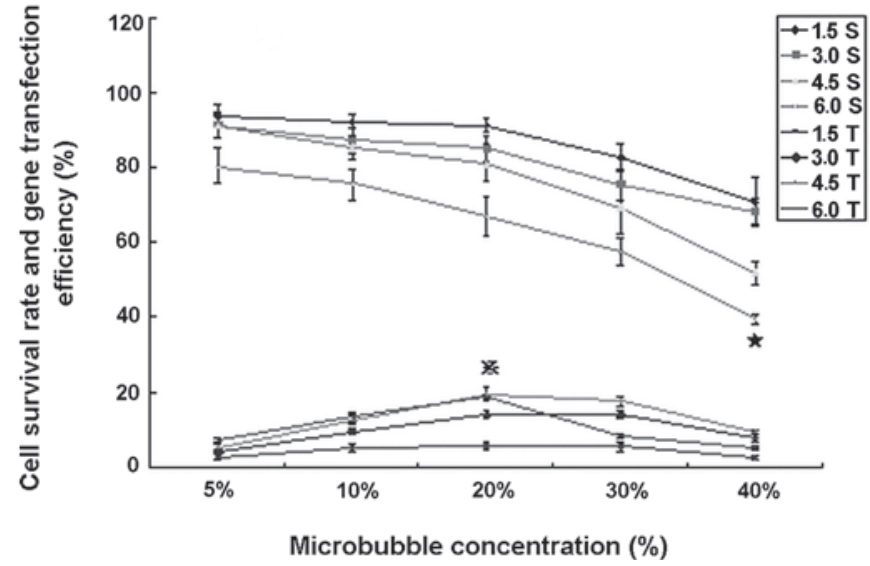

Figure 1. Gene delivery efficiency and cell viability under various microbubble concentrations and ultrasonic irradiation doses of 1.5, 3.0, 4.5 and $6.0 \mathrm{~W} / \mathrm{cm}^{2} \mathrm{x}$ sec. Ultrasonic irradiation doses were 1.5, 3.0, 4.5 and $6.0 \mathrm{~W} / \mathrm{cm}^{2} \mathrm{x}$ sec. Gene transfection efficiency was relatively low, especially at 5 and $40 \%$ microbubble concentration. Cell viability was kept within a safe range with the exception of $40 \%$ microbubble concentration $(\mathrm{P}<0.001)$. $\star$, cell survival rate was significantly lower than that of any other microbubble concentration $(\mathrm{P}<0.001)$; $\star$, gene transfection efficiency under $4.5 \mathrm{~W} / \mathrm{cm}^{2} \mathrm{x} \mathrm{sec}$ was significantly higher than that in any other conditions, with the exception of $6.0 \mathrm{~W} / \mathrm{cm}^{2} \mathrm{x} \mathrm{sec}$ at $20 \%$ microbubble concentration $(\mathrm{P}<0.001)$; however, the latter cell survival rate was severely decreased $(\mathrm{P}<0.001) .1 .5 \mathrm{~S}, 3.0 \mathrm{~S}, 4.5 \mathrm{~S}$ and $6.0 \mathrm{~S}$, cell survival rate at $1.5,3.0,4.5$ and $6.0 \mathrm{~W} / \mathrm{cm}^{2} \mathrm{x} \mathrm{sec}$, respectively; $1.5 \mathrm{~T}, 3.0 \mathrm{~T}, 4.5 \mathrm{~T}$ and $6.0 \mathrm{~T}$, gene transfection efficiency under $1.5,3.0,4.5$ and $6.0 \mathrm{~W} / \mathrm{cm}^{2} \mathrm{x} \mathrm{sec}$, respectively.

Microbubble concentration. Gene delivery efficiency was relatively low at a microbubble concentration of 5 and $10 \%$ $(\mu 1 / \mu 1)$, efficiency increased significantly at 20 and $30 \%$ concentrations under $3.0 \mathrm{~W} / \mathrm{cm}^{2} \mathrm{x} \mathrm{sec}$, reaching the highest level at $4.5 \mathrm{~W} / \mathrm{cm}^{2} \mathrm{x}$ sec. No significant differences were identified with respect to gene delivery efficiency between these two microbubble concentrations under the ultrasound irradiation of $3.0 \mathrm{~W} / \mathrm{cm}^{2} \mathrm{x} \mathrm{sec}(14.04 \pm 1.04 \mathrm{vs} .13 .92 \pm 0.97 \%$, $\mathrm{P}>0.05)$ and $4.5 \mathrm{~W} / \mathrm{cm}^{2} \mathrm{x}$ sec $(19.66 \pm 1.67$ vs. $17.54 \pm 1.41 \%$, $\mathrm{P}>0.05)$. However, the gene transfection efficiency was significantly decreased at $40 \%$ microbubble concentration under any ultrasound exposure condition $(\mathrm{P}<0.01)$. Cell viability was significantly decreased at microbubble concentrations
$>30 \%$ ( $\mathrm{P}<0.01$; Fig. 1). Therefore, $20 \%$ microbubble concentration was determined as optimal for experiments.

DNA concentration. Gene transfection efficiency was low at DNA concentrations of $2.5 \mu \mathrm{g} / \mathrm{ml}(1.08 \pm 0.35 \%)$ and $5 \mu \mathrm{g} / \mathrm{ml}$ $(3.98 \pm 1.13 \%)$; it increased significantly at DNA concentrations of $10 \mu \mathrm{g} / \mathrm{ml}(9.14 \pm 1.56 \%)$ under ultrasound irradiation of $1.5 \mathrm{~W} / \mathrm{cm}^{2}$ and $30 \mathrm{sec}$ (ultrasound exposure dose, $4.5 \mathrm{~W} / \mathrm{cm}^{2} \mathrm{x} \mathrm{sec}$ ) with $20 \%$ microbubble concentration. Thereafter, the efficiency reached a plateau level at DNA concentrations of 15, 20 and $30 \mu \mathrm{g} / \mathrm{ml}$. No significant differences were identified among the above concentrations $(18.74 \pm 1.57,18.52 \pm 1.29$ and $19.02 \pm 2.10$, respectively, all $\mathrm{P}>0.05)$. No significant differences were identified in cell viability between the various DNA concentrations (Fig. 2). In our experiments, $15 \mu \mathrm{g} / \mathrm{ml}$ was determined to be the optimal plasmid concentration.

$F B S$. No significant differences were identified in gene transfection efficiency between the group containing $8 \%$ FBS and the group in its absence $(19.2 \pm 1.1$ vs. $19.7 \pm 0.9 \%, \mathrm{P}>0.05)$. In addition, no significant differences in cell viability were identified between the two groups $(86.3 \pm 2.1$ vs. $86.6 \pm 1.7 \%$, $\mathrm{P}>0.05$; results not shown).

Mixing mode of cell and microbubble. The gene transfection efficiency was $8-15 \%$ under the cell adherence mode (10.4 $1.2 \%)$; however, the cell survival rate was significantly decreased following ultrasound irradiation $(65.4 \pm 2.9 \%)$. Comparatively, gene delivery efficiency reached almost $20 \%(19.7 \pm 0.7 \%, \mathrm{P}<0.01)$ under cell suspension mode without a significant decrease in cell viability $(76.6 \pm 2.1 \%, \mathrm{P}<0.01)$. Therefore, the cell suspension mode was the optimal mode in our experiments.

Integrity of hAng-1 DNA. Agarose gel electrophoresis revealed a band corresponding to the hAng-1 gene that underwent ultrasound irradiation at the same position as a band produced for the hAng-1 plasmid which had not undergone ultrasound irradiation. This indicates that ultrasound irradiation used in this experiment did not cause the hAng-1 gene DNA chain to break, and therefore did not affect integrity (Fig. 3).

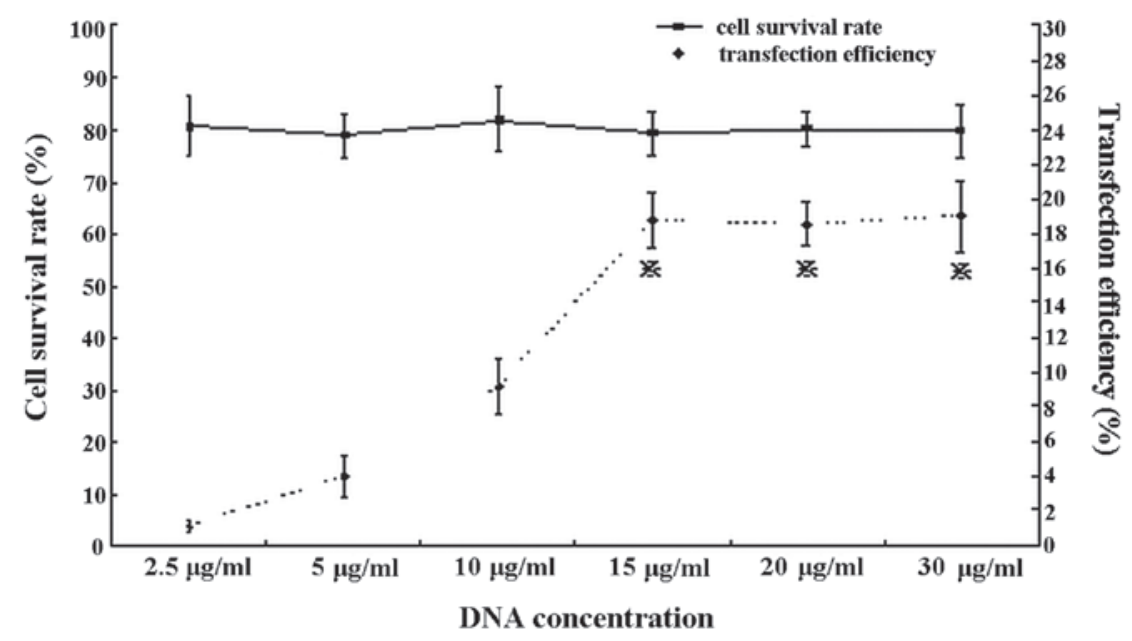

Figure 2. Gene delivery efficiency and cell viability under various DNA concentrations of 2.5, 5, 10, 15, 20 and 30 $\mu \mathrm{g} / \mathrm{ml}$. Ultrasound exposure intensity was $1.5 \mathrm{~W} / \mathrm{cm}^{2}$ and $30 \mathrm{sec}$, microbubble concentration was $20 \%$. $※$, gene transfection efficiency was significantly higher than $2.5,5$ and $10 \mu \mathrm{g} / \mathrm{ml}(\mathrm{P}<0.01)$. 


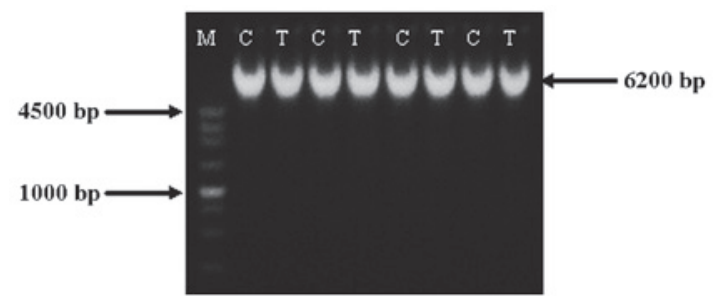

Figure 3. eGFP-C3-hAng-1 plasmid electrophoresis result. M, DNA marker; $\mathrm{C}$, plasmid band without ultrasound irradiation; T, plasmid band after ultrasound irradiation. The size and length of bands $\mathrm{C}$ and $\mathrm{T}$ were the same, indicating that ultrasound irradiation does not affect plasmid integrity.

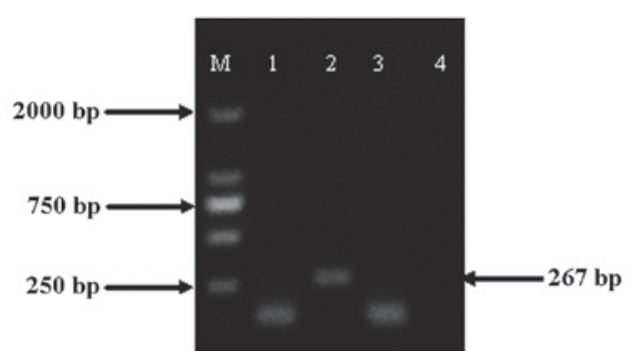

Figure 4. hAng-1 mRNA expression detected by RT-PCR analysis. M, DNA marker; band 2, ultrasound/microbubble-mediated group; band 1, $\beta$-actin of this group; band 4 , control group; band $3, \beta$-actin of control group.

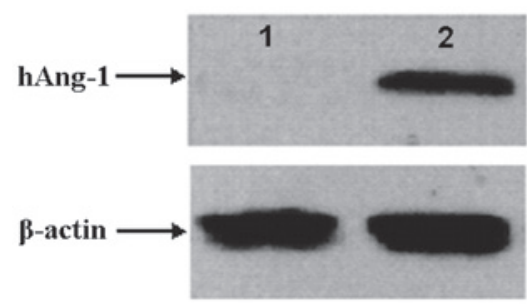

Figure 5. hAng-1 protein analysis by western blot analysis. 1, control group; 2 , ultrasound/microbubble-mediated group. Protein expression was detected in ultrasound/microbubble-treated cells (2) and no protein expression was detected in the control cells (1).

Determination of mRNA of hAng-1 gene. Following 48-h transfection, there was marked eGFP-C3-hAng-1 mRNA expression in the ultrasound/microbubble-mediated gene delivery group compared with the control group, demonstrating that the eGFP-C3-hAng-1 gene is expressed via the ultrasound/microbubble-mediated method (Fig. 4).

Determination of hAng-1 protein.hAng-1 protein was detected using SDS-PAGE/western blot analysis in the ultrasound/ microbubble-mediated gene delivery group but was absent in the control group. This implied that the delivery process via ultrasound/microbubbles does not affect the gene eGFPC3-hAng-1a translation into the functional protein (Fig. 5).

\section{Discussion}

Optimal gene transfection conditions are utilized to enhance gene expression levels and restrict cell death to $5 \%$ or less (11). Previous studies have discussed the effect of ultrasound exposure and irradiation intensity on gene delivery $(12,13)$; however, the influential factors are more extensive than discussed. Contrast agents, target cell and tissue type, corpuscular physiological status and uptake capacity of cells to DNA, etc., are the most common factors affecting the efficiency of gene transfer (14). Determining how to match these parameters to achieve optimal conditions is critical for the improvement of gene delivery and expression.

The present study aimed to investigate hAng-1 gene delivery efficiency and cellular viability under various conditions. The study mainly focused on contrast agent, DNA concentration, mixing mode of microbubble and cells and the presence of FBS. With regard to contrast agent, we identified that when the SonoVue microbubble was used as a gene transport medium, with a gradual increase in ultrasound irradiation intensity to $1.5 \mathrm{~W} / \mathrm{cm}^{2}$ and exposure time to $30 \mathrm{sec}$, and a microbubble concentration of $20 \%$, a progressive increase in hAng-1 gene transfer was observed. Cell viability was gradually decreased, although it remained at an acceptable range. The above three parameters had similar effects on gene delivery and cell viability. However, hAng-1 gene delivery did not continue to rise with continued increase of levels of the above parameters. This indicates that for a certain type of cell or tissue, particular gene transfection efficiency cannot be increased after reaching saturated levels. Beyond this level, the gene transfection efficiency decreased rather than increased, likely due to extensive cell death. Under these conditions, the ultrasound/microbubble gene delivery may become unsafe. It should be noted that under conditions of transfection saturation, a small increase in ultrasound exposure or the microbubble concentration improves gene transfection minimally, and at the cost of a large number of cell deaths. Therefore, to obtain the optimal transfection parameters, instead of maximal transfection efficiency, we should select lower levels of ultrasound radiation intensity, exposure duration and microbubble concentration within the plateau phase parameters to achieve higher transfection efficiency and ensure the safety of ultrasound/microbubble as a gene carrier.

In addition, we revealed that the mixed mode of microbubbles and cells also affected transfection efficiency. In preliminary experiments, we observed that in order to obtain high transfection efficiency, cell and microbubble contact must be sufficient. Thus, we added the microbubble solution with a concentration of $20 \%$ microbubble solution per $\mu 1$ into the adherent cells in a 6-well plate after removing the culture medium, and then performed ultrasound irradiation. Although this method improved the transfection efficiency, it reduced cell viability notably and generated unstable results and poor reproducibility. The causes may be: i) the microbubble always floats on the surface of the transfection system due to buoyancy effects, thus it cannot be in contact with adherent cells, ultimately decreasing the effects of the microbubble on cells; ii) ultrasonic irradiation causes detachment of adherent cells from the bottom of the 6-well plate, resulting in reduced cell activity. Therefore, we mixed the concentrated cell suspension with the microbubble solution. We identified that this method not only increased contact opportunity between microbubbles and cells, but also reduced the negative effects on cell activity, thereby, significantly increasing Ang-1 gene transfection and decreasing cell injury.

Previous studies used fresh culture medium without serum during gene transfection, changing to culture medium 
containing serum post-transfection during the in vitro ultrasound/microbubble gene delivery experiment (15-17). The ultimate aim of the present study was to apply this new gene delivery system in vivo, therefore it was necessary to investigate the system in the presence of FBS, to determine whether the microbubble/ultrasound-mediated gene transfer system performed an effective gene transfection. The results of this study indicate that the presence of FBS has no effects on the hAng-1 gene transfection efficiency and cell viability. The therapeutic gene was previously reported to be administered by intravenous injection, therefore, successful transfection of the plasmid DNA in vitro in the presence of FBS may be of greater value for application of the ultrasound-directed gene transfer to cardiovascular disease therapy in vivo.

Successful gene transfection not only requires cells to take up the exogenous gene, but also maintain normal function of expression and translation of the ingested gene. The heat and micro-jet generated by cavitation effects may cause the plasmid DNA to fragment $(18,19)$, endanger the integrity of DNA and affect its normal expression and functions. Therefore, we investigated DNA integrity under optimal transfection conditions. We identified that under optimal conditions, the ultrasound/microbubble-mediated gene transfection system did not affect DNA integrity in vitro. Furthermore, detection of hAng-1 mRNA by quantitative RT-PCR and hAng-1 protein by SDS-PAGE/western blot analysis provided evidence that the transferred hAng-1 gene was expressed, and suggested normal function inside the cells.

Current studies demonstrate relatively low transfection efficiency, which is a common challenge for all non-viral gene delivery methods $(20,21)$. The main causes may be correlated with lack of effective protection of the plasmid when entering the cytoplasm and possible degradation of the plasmid by DNase in the cytoplasm before entering the nucleus (22). It is vital to successfully optimize transfection parameters of the hAng-1 gene as an angiogenesis gene for cardiac ischemic diseases therapy in humans, including thorough understanding of the effects of the system on blood flow and ultrasound energy attenuation following penetration of skin and muscle tissue (23). The above optimal parameters function as a significant reference, but currently remain unsuitable for experiments in vivo.

To summarize, for a particular type of contrast agent, the exogenous gene, and the target tissue or cell, it is necessary to determine the specific optimal transfection conditions for utilization of the ultrasound/microbubble gene transfer system. Gene transfection efficiency is determined by multiple factors and interactions. Further studies are required to clarify the biological effects and functional mechanisms.

\section{Acknowledgements}

This study was supported by a grant from the National Natural Science Fundation of China (30600141).

\section{References}

1. Korpanty G, Chen S, Shohet RV, et al: Targeting of VEGFmediated angiogenesis to rat myocardium using ultrasonic destruction of microbubbles. Gene Ther 12: 1305-1312, 2005.
2. Inagaki H, Suzuki J, Ogawa M, Taniyama Y, Morishita R and Isobe M: Ultrasound-microbubble-mediated NF-kappaB decoy transfection attenuates neointimal formation after arterial injury in mice. J Vasc Res 43: 12-18, 2006.

3. Yamashita T, Sonoda S, Suzuki R, et al: A novel bubble liposome and ultrasound-mediated gene transfer to ocular surface: RC-1 cells in vitro and conjunctiva in vivo. Exp Eye Res 85: 741-748, 2007.

4. Shen ZP, Brayman AA, Chen L and Miao CH: Ultrasound with microbubbles enhances gene expression of plasmid DNA in the liver via intraportal delivery. Gene Ther 15: 1147-1155, 2008.

5. Larina IV, Evers BM and Esenaliev RO: Optimal drug and gene delivery in cancer cells by ultrasound-induced cavitation. Anticancer Res 25: 149-156, 2005.

6. Duvshani-Eshet M, Adam D and Machluf M: The effects of albumin-coated microbubbles in DNA delivery mediated by therapeutic ultrasound. J Control Release 112: 156-166, 2006.

7. Nie F, Xu HX, Tang Q and Lu MD: Microbubble-enhanced ultrasound exposure improves gene transfer in vascular endothelial cells. World J Gastroenterol 12: 7508-7513, 2006.

8. Taniyama Y, Tachibana K, Hiraoka K, et al: Development of safe and efficient novel nonviral gene transfer using ultrasound: enhancement of transfection efficiency of naked plasmid DNA in skeletal muscle. Gene Ther 9: 372-380, 2002.

9. Guo DP, Li XY, Sun P, et al: Ultrasound/microbubble enhances foreign gene expression in ECV304 cells and murine myocardium. Acta Biochim Biophys Sin 36: 824-831, 2004.

10. Li T, Tachibana K, Kuroki M and Kuroki M: Gene transfer with echo-enhanced contrast agents: comparison between Albunex Optison and Levovist in mice - initial results. Radiology 229: 423-428, 2003.

11. Miller DL, Dou C and Song J: DNA transfer and cell killing in epidermoid cells by diagnostic ultrasound activation of contrast agent gas bodies in vitro. Ultrasound Med Biol 29: 601-607, 2003.

12. Li YS, Davidson E, Reid CN and McHale AP: Optimising ultrasound-mediated gene transfer (sonoporation) in vitro and prolonged expression of a transgene in vivo: potential applications for gene therapy of cancer. Cancer Lett 273: 62-69, 2009.

13. Kinoshita M and Hynynen K: Key factors that affect sonoporation efficiency in in vitro settings: the importance of standing wave in sonoporation. Biochem Biophys Res Commun 359: 860-865, 2007.

14. Suzuki R, Takizawa T, Negishi Y, Utoguchi N and Maruyama K: Effective gene delivery with novel liposomal bubbles and ultrasonic destruction technology. Int J Pharm 354: 49-55, 2008.

15. Rahim A, Taylor SL, Bush NL, ter Haar GR, Bamber JC and Porter CD: Physical parameters affecting ultrasound/microbubble-mediated gene delivery efficiency in vitro. Ultrasound Med Biol 32: 1269-1279, 2006.

16. Taniyama Y, Tachibana K, Hiraoka K, et al: Development of safe and efficient novel nonviral gene transfer using ultrasound: enhancement of transfection efficiency of naked plasmid DNA in skeletal muscle. Circulation 105: 1233-1239, 2002.

17. Guo DP, Li XY, Sun P, et al: Ultrasound-targeted microbubble destruction improves the low density lipoprotein receptor gene expression in HepG2 cells. Biochem Biophys Res Commun 343: 470-474, 2006.

18. O'Brien WD Jr: Ultrasound-biophysics mechanisms. Prog Biophys Mol Biol 93: 212-255, 2007.

19. Kimmel E: Cavitation bioeffects. Crit Rev Biomed Eng 34: 105-161, 2006.

20. Glover DJ, Lipps HJ and Jans DA: Towards safe non-viral therapeutic gene expression in humans. Nat Rev Genet 6: 299-310, 2005.

21. Hurtado Picó A, Wang X, Sipo I, et al: Viral and nonviral factors causing nonspecific replication of tumor- and tissue-specific promoter-dependent oncolytic adenoviruses. Mol Ther 11: 245-256, 2005

22. Mehier-Humbert S, Bettinger T, Yan F and Guy RH: Ultrasoundmediated gene delivery: Kinetics of plasmid internalization and gene expression. J Control Release 104: 203-211, 2005.

23. Suzuki R, Takizawa T, Negishi Y, et al: Tumor specific ultrasound enhanced gene transfer in vivo with novel liposomal bubbles. J Control Release 125: 137-144, 2008. 